\title{
Interculturalidad crítica, teoría sociolingüística e igualdad de oportunidades. La extraordinaria historia de un menor infractor
}

\author{
Critical interculturality, socio-linguistic theory and equity. The extraordinary story of a \\ young offender
}

\author{
David Herrera Pastor \\ e-mail: dvherrera@uma.es \\ Universidad de Málaga. España \\ Caterí Soler García iD \\ e-mail: caterisolergarcia@uma.es \\ Universidad de Málaga. España \\ Iulia Mancila (iD) \\ e-mail: imancil@uma.es \\ Universidad de Málaga. España
}

\section{Resumen}

En este artículo se analiza cómo las personas que nacen y se educan en contextos desfavorecidos se socializan en una cultura y unos códigos que limitan sus posibilidades de desarrollo. Para profundizar sobre dicha cuestión consideramos un fragmento de la historia de vida de Samir Almasi ${ }^{1}$, un menor infractor que se convirtió en educador de menores tras cumplir un proceso de reforma juvenil. Una de las claves para que se produjese aquel extraordinario cambio fue que durante su infancia había adquirido una cultura que le permitía desenvolverse adecuadamente en cualquier entorno, no sólo en su contexto de origen. Aquella situación resultaba llamativa, porque la mayoría de sus compañeros en el centro de internamiento de menores infractores, no pudieron evolucionar tan favorablemente como él porque manejaban unos lenguajes de interacción, sobre todo comportamentales, muy alejados de los demandados por la sociedad. Hacemos una analogía de esa situación utilizando los códigos restringidos de la teoría socio-lingüística de Bernstein. Los resultados muestran que las personas que han experimentado una socialización limitante sufren una situación determinista que dificulta su emancipación fuera de su ambiente primario. Desde una perspectiva crítica intercultural se reivindica la de-construcción de las causas que generan esa situación y garantizar el desarrollo cultural de todas las personas, fundamentalmente, quienes proceden de entornos deprimidos culturalmente, en pos de una verdadera igualdad de oportunidades y de una sociedad inclusiva; ello debe ser una de las pretensiones de la educación obligatoria y pública.

Palabras clave: Interculturalidad crítica; socialización; determinismo; Teoría de los códigos sociolingüísticos; educación obligatoria; igualdad de oportunidades.

\begin{abstract}
This article discusses how people raised in disadvantaged contexts are socialized by specific cultural codes that limit their development. To illustrate it, a fragment of Samir Almasi's life history is presented. He was a minor offender who became an educator after a process of juvenile justice reform. One of the fundamental key related to this extraordinary change was the fact that during his childhood he had acquired a culture that allowed him to function adequately in any social environment, not only in his context of origin. Most of his colleagues in the centre of internment of juvenile offenders could not react and develop alike because they were socialized in specific languages of interaction, and behaviour not accepted by the majoritarian society. We make an analogy of that situation using the "restricted codes" notion from Bernstein's socio-linguistic theory. The results show that people who has experienced a culturally limited socialization makes emancipation unreachable outside of their primary environment. From a critical intercultural perspective, we argue that it is necessary to analyse the determinant factors of this unequal situation. Likewise, we argue for the acquisition of a minimum culture for all people, fundamentally, those from culturally depressed environments, in pursuit of a true equal opportunities and an inclusive society. The public education must be a guarantee of this cultural right.

Keywords: Critical interculturality; socialization; determinism; Critical sociolinguistic theory; compulsory education; equality.

Recibido / Received: 15-03-2018

Aceptado / Accepted: 16-07-2018

Cómo referenciar este artículo / How to reference this article:

Herrera Pastor, D., Soler García, C., \& Mancila, I. (2019). Interculturalidad crítica, teoría sociolingüística e igualdad de oportunidades. La extraordinaria historia de un menor infractor. Tendencias Pedagógicas, 33, 69-82. doi: $10.15366 /$ tp2018.32.006
\end{abstract}

${ }^{1}$ Samir Almasi es un pseudónimo para garantizar el anonimato del protagonista. 


\section{Introducción}

En el presente artículo se discute sobre cómo las diferencias culturales, con respecto a la cultura hegemónica, limitan el desarrollo de las personas, fundamentalmente, de las que se encuentran en los estratos más bajos de la sociedad.

Con la intención de profundizar sobre dicha cuestión tomamos como fuente de análisis un fragmento de la investigación llevada a cabo en torno a la historia de Samir Almasi (Herrera y De Oña, 2016 y 2017), un menor infractor que pudo transformar sustancialmente su vida a través de un proceso de reforma juvenil, tanto que se convirtió en educador de menores. Una de las claves primordiales para que aquel cambio tuviese lugar fue que manejaba los códigos de la cultura dominante.

La tesis principal de la teoría de los códigos sociolingüísticos de Bernstein $(1988,1989)$, que examina la relación entre lenguaje, socialización y clase social, será tomada en consideración para el estudio de esa situación. El lenguaje no se interpretará desde un punto de vista, únicamente, idiomático, sino desde una perspectiva más amplia, entendiendo como tal cualquier forma de interacción semiótica.

En esta investigación se utilizó una metodología biográfica, materializada en formato de historia de vida. Para construir la biografía de Samir, y posteriormente analizarla, se utilizaron numerosas fuentes de información y estrategias de recolección de datos. Entre las más destacadas, las entrevistas biográficas al protagonista de la historia y a doce informantes clave [la jueza que llevó su caso, el subdirector del centro de internamiento de menores infractores (CIMI) en el que estuvo, su tutor en el CIMI, la tutora de libertad vigilada, su madre de acogida, su hermano biológico, amigos,...]. También se recabó información de diversas fuentes documentales: el expediente de Samir en el juzgado de menores y su homólogo en la Dirección General de Reforma Juvenil (que es el órgano de la Junta de Andalucía encargado de materializar las medidas judiciales). Y se contó con más de doscientas fotografías, la mayoría de ellas de su experiencia en el centro de internamiento.

El relato biográfico se materializó de acuerdo al modelo de relatos cruzados (Lewis, 1973; Gamella, 1990) de manera no consecutiva, por lo que se armó, de manera paralela, a partir del cruce de los distintos testimonios y registros recabados (Herrera, 2016; Herrera, Mancila y Kushner, 2017). Y el análisis se llevó a cabo de manera inductiva, a partir de la emergencia de datos sustantivos relacionados con el foco de la investigación.

Se eligió dicha metodología, por diversos motivos, entre ellos, porque es muy adecuada para el estudio de casos minoritarios y porque posee una idiosincrasia que otorga un plus a la investigación en términos de ética, política, pedagogía social y visión holística e integral.

Los datos muestran que, a pesar de que Samir era un joven procedente de otro país y de un entorno deprimido, poseía un sustrato socializador y unos códigos de interacción que le permitían salir airoso de las demandas del sistema de justicia. A lo largo de su proceso de reforma juvenil, cuando era necesario, utilizaba los elementos culturales oportunos (expresiones verbales, comportamientos, hábitos, etc.) para responder adecuadamente a lo que se le requería, lo que posibilitó una evolución muy favorable de su caso.

Samir destacaba porque sus lenguajes, fundamentalmente conductuales, nada tenían que ver con los de la mayoría de menores infractores que tienen cumplir algún tipo de proceso de reforma juvenil. Eso quedó patente, sobre todo, en el centro de internamiento. El grueso de esos menores suele manejar unos códigos de interacción que habitualmente se encuentran alejados de los aceptados socialmente por el conjunto de la población. La distancia entre la microcultura, no sólo de los menores infractores, sino de quienes se encuentran en los estratos sociales más desfavorecidos ( $y$ poseen esas culturas minoritarias y manejan esos códigos alejados), y la cultura hegemónica suele coartar su desarrollo laboral, económico, social, etc. Esa distancia provoca reproducción social, en cuanto que apenas concede margen de desarrollo y promoción a las personas que se encuentran en 
esas capas sociales.

En virtud de ello, se requiere una auténtica interculturalidad crítica y mecanismos que modifiquen las dinámicas actuales (segregadoras, hegemonicistas, etc.) (Leiva, 2015 y 2013) para fomentar la interacción entre las culturas, particularmente, entre las más alejadas con la cultura dominante, sin que esta última las aplaste, absorba, etc. De modo que se establezca un diálogo entre ellas en pos de la diversidad, la inclusión y la igualdad de oportunidades, que rompa con las dinámicas deterministas.

Estas ideas necesitan articularse en distintas acciones, algunas de nuestras propuestas al respecto son: Por un lado, garantizar el desarrollo cultural a quienes viven en esos mundos simbólicos más limitados, para contrarrestar o compensar esa situación y que puedan desenvolverse adecuadamente en cualquier escenario, no sólo en su contexto más cercano. Esa labor pedagógica la debe garantizar el sistema educativo obligatorio.

Por otro lado, se ha de hacer una labor pedagógica con el conjunto de la sociedad (fundamentalmente, con la mayoría perteneciente a la cultura hegemónica) para romper estereotipos y tender puentes, haciendo comprender que las personas que provienen de estratos socio-culturales deprimidos, se han criado en un entorno hostil y, como consecuencia, han desarrollado un sustrato socializador y unos códigos determinados de conducta, expresión, raciocinio, etc., producto de su vida en ese entorno. De ese modo se posibilitaría la comprensión y puesta en práctica de una interculturalidad libre y plena.

Por último, se han de desmontar las dinámicas del sistema social que perpetúan a las minorías culturales en su situación y les impiden promocionar socialmente.

\section{Fundamentación teórica}

En este apartado se presenta un breve marco conceptual de los preceptos epistemológicos fundamentales que se toman en consideración para interpretar e indagar la realidad sujeta a estudio. En concreto, esta fundamentación teórica se realiza en torno a dos ideas: la primera, sobre el concepto de socialización, que es esencial para la comprensión del uso de los códigos sociolingüísticos y, la segunda, sobre la teoría de los códigos sociolingüísticos, cuya tesis principal comprende la esencia del análisis que se va a realizar.

\subsection{Socialización: patrón cultural básico que mediatiza los códigos sociolingüísticos del sujeto.}

La socialización es el proceso de culturización de la persona en su sentido más elemental. Cada persona desarrolla la capacidad simbólica y recrea el conjunto de significados que le envuelve (que son contingentes al con-texto ${ }^{2}$ en el que se mueve) a partir de la interacción con los distintos agentes sociales con los que se relaciona.

López (2013), por su parte, afirma que "somos convivencia” y que "socialización significa el proceso de concretarse en lo social” (p. 189). La socialización es la "iniciación en los valores, costumbres, prácticas, hábitos e instituciones que conforman de modo característico la cultura humana3" (Carr, 2005, p. 21). Aunque también se puede conseguir con otro tipo de procesos pedagógicos, "no se puede entender la educación sin una referencia específica a la socialización (...), entendida como un conjunto de hábitos y actitudes directamente vinculadas con la vida de relación" (Sarramona, 2008, p. 219). Es un proceso que se inicia como adaptación a la vida social humana, se suele desarrollar de manera natural y acrítica (socialización primaria) y a través del cual el sujeto se

\footnotetext{
${ }^{2}$ Con-texto: Escenario con significación.

${ }^{3}$ La socialización es, además, es un proceso de humanización, ya que en el proceso de socialización es donde el homínido se hace humano. ¿Por qué no se le llama, entonces, proceso de culturización o proceso de humanización? Porque por encima de todo, somos seres sociales, nos hacemos humanos en sociedad. En palabras de Durkheim (1976) "es posible deducir a qué se reduciría el hombre si se le retirase todo lo que recibe de la sociedad: volvería a caer en el nivel en que se mueven los animales" (p. 103).
} 
conforma en virtud de su entorno social. Por eso, la cualidad de la socialización depende en gran medida de las características del entorno y del mundo simbólico en el que se encuentre la persona.

La socialización que se desarrolla durante la infancia y adolescencia, que son estadios evolutivos tan importantes en el desarrollo de la persona, resultan fundamentales en la configuración del patrón personal del sujeto. La cultura experiencial que se desarrolle durante ese periodo mediatizará la capacidad semiótica del individuo en todos los sentidos: lingüístico, cognitivo, social, afectivo, moral, etc. También tendrá una influencia trascendental en el desarrollo de su identidad personal.

\subsection{Teoría de los Códigos Sociolingüisticos y entornos socioculturamente deprimidos.}

El profesor Basil Bernstein fue un importante sociólogo que, entre otras cosas, estudió, de manera exhaustiva, la relación entre semiótica, poder y control de la población $(1990,1998)$. En este subapartado nos vamos a interesar por la teoría de los Códigos Sociolingüísticos, con la que profundizó sobre la interrelación existente entre clase social, socialización y lenguaje $(1988,1989)$, que son los elementos que se analizan en este artículo.

Bernstein terminó aterrizando su teoría en el ámbito escolástico o de la educación formal. Sin embargo, nosotros vamos a aproximarnos a esos conceptos desde el ámbito social, por lo que nos acercaremos a ellos desde una perspectiva intercultural en un sentido más amplio.

Y es que no todos los grupos sociales acceden a la cultura en el mismo grado. En el subapartado anterior ya se ha indicado que los contextos en los que las personas crecen y se desarrollan, fundamentalmente en los estadios evolutivos primigenios, tienen una trascendencia esencial en el desarrollo de sus miembros. La socialización primaria que se experimenta en estratos sociales deprimidos suele tener lugar en un ambiente de cultura muy limitada, en cuanto que no se tiene contacto con muchos de los códigos o lenguajes de la cultura dominante. Eso suele limitar las posibilidades de desarrollo de las personas que proceden de esos contextos, que no poseen las herramientas culturales necesarias (no sólo lingüísticas, sino también, actitudinales, comportamentales, etc. ${ }^{4}$, lo que les dificulta salir adelante fuera de su entorno. En buena medida, esa limitación cultural delimita su campo de acción al entorno con el que se encuentra familiarizado, coarta otras posibilidades de emancipación y predestina hacia la reproducción social.

Los dos conceptos más conocidos de la citada teoría de Bernstein: código restringido y código elaborado, nos permiten (fundamentalmente el primero) hacer un análisis de esa situación desde una perspectiva crítica. Londoño y Castañeda (2011) entienden que "El código restringido se caracteriza por girar alrededor de significados dependientes del contexto, conceptos particulares y locales, mientras que el código elaborado sobresale por sus significados más independientes del contexto, conceptos universales y menos locales" (p. 22). En este trabajo entendemos los códigos desde su perspectiva semántica holística, más que sintáctica o léxica (Poveda, 2001). En los entornos desfavorecidos (Especialmente, en los contextos de exclusión donde se suele desarrollar una microcultura propia), la cultura que interiorizan sus habitantes a través de los procesos de socialización resulta insuficiente y/o inadecuada para satisfacer apropiadamente las demandas que se les plantean en contextos donde prevalece la cultura de la mayoría (la cultura dominante), por lo que estaríamos postulando que esa población poseería un código restringido (grupos desfavorecidos - subcultura o cultura marginal).

El funcionamiento y las estructuras del sistema social se rigen conforme a esa cultura mayoritaria, por lo que, quienes proceden de entornos minoritarios suelen encontrarse en situación de desventaja en esos contextos. Las personas que poseen restringidos sus códigos culturales se suelen encontrar alienados fuera de su hábitat, por lo que no se puede decir que puedan desarrollarse con las mismas oportunidades (oportunidades equivalentes) en escenarios que se rigen por los códigos y lenguajes de la cultura dominante. Por tanto, la relación dialéctica sujeto-estructura social, el lugar que el sujeto ocupa en el entramado social, y la enculturización que experimenta de partida

\footnotetext{
${ }^{4}$ Maturana (1997) se referiría a ello como lenguajear. 
(socialización y lenguaje) condiciona, claramente, sus posibilidades de desarrollo. Luego, la transmisión de unos códigos culturales que contribuyen a la reproducción cultural y simbólica y legitimación de la estructura social dominante (que se hace hegemónica), limitando, cuando no excluyendo, a todos aquellos individuos que no comparten dichos códigos, facilita nuevas perspectivas de análisis de la llamada "interculturalidad", aportando herramientas clave para comprender por qué ciertos individuos llegan a tener éxito en sus trayectorias socio-educativas y otros no, como es el caso de Samir Almasi, protagonista de la investigación que se presenta en este artículo.

Debe quedar claro, que "ninguno de los dos códigos es más importante que el otro, puesto que los dos les permiten a los hablantes poder interactuar con su entorno y satisfacer las necesidades de comunicación" (Londoño y Castañeda, 2011, p. 22). Cada código (cada lenguaje) permite desenvolverse adecuadamente en un contexto determinado.

No obstante, hacer "bilingües" a las personas en ese sentido, es decir culturalmente hábiles, debe ser uno de los objetivos del sistema educativo, para que puedan tener mayores oportunidades de desarrollo y emancipación. La institución educativa debe garantizar que toda la población escolar, sin distinción, experimente un proceso de socialización en la cultura hegemónica, además de en su microcultura particular. Dicho de otro modo, respetando su identidad individual y colectiva, se ha de tratar de acercar a las personas con carencias culturales al mundo simbólico dominante en el espacio social general, para que puedan tener otras posibilidades de desarrollo, de modo que se les puedan abrir horizontes y oportunidades de emancipación en otros escenarios.

\section{Síntesis de la biografía}

Para que el lector pueda contextualizar y comprender adecuadamente las discusiones e ideas que se plantean en los siguientes apartados, realizamos una breve síntesis de la biografía del protagonista de la historia de vida eje de este artículo:

Samir nació en el seno de una familia muy numerosa (diez, hijos) del norte de Marruecos. Vivía en un entorno semi-rural en el que las posibilidades de desarrollo pasaban por reproducir las pautas de vida de sus progenitores y hermanos mayores: trabajos no cualificados, jornadas infinitas y remuneración muy baja, unido a escasas coberturas laborales y sociales. Con 13 años abandonó la escuela y empezó a trabajar en un bar en el que tenía que echar alrededor de 12 horas cada jornada para ganar un euro al día. Tras varios años errando por empleos precarios, empezó a darse cuenta de que habia personas que gozaban de unas condiciones de vida muy diferentes, muy acomodadas. Además, recibía enormes influjos, fundamentalmente a través de los medios de comunicación, sobre el estilo de vida occidental que parecía estar muy consolidado. Un día montó en una barca cargada de bachís y cruzó el Estrecho de Gibraltar. Por la operación le reportaron 6000 euros. A partir de entonces empezó a trabajar para aquella organización desde las costas del sur de España (organizando desembarcos y distribuyendo mercancía). Después de algo más de un año realizando ese trabajo fue detenido por la policia. Como todavía era menor de edad (tenía 17 años) se le condenó a un proceso de reforma juvenil de veinticuatro meses que se dividió en dos medidas: la primera, más extensa, de internamiento en centro, y la segunda, de libertad vigilada. Samir no era un delincuente, sino un joven que decidió migrar para cambiar de vida y se involucró en aquella mafia para tratar de lograr su objetivo. Como consecuencia, se adaptó muy bien al proceso de reforma que tuvo que cumplir, y progresó adecuadamente durante dicho proceso. Tanto, que finalizándolo (hace abora, aproximadamente, tres lustros) le ofrecieron la oportunidad de empezar a trabajar como monitor en un piso de protección de menores. Aceptó el ofrecimiento y años después emperó a desempeñar labores de educador. Aquel trabajo le permitió estabilizarse definitivamente en aquella ciudad y conseguir su propósito: mejorar sus condiciones y posibilidades de vida ${ }^{5}$.

\section{Metodología}

La metodología de investigación biográfica fue la que se utilizó para la realización del trabajo. Se eligió porque era la que mejor se adecuaba a las necesidades de la investigación y porque se trata de una metodología que posee una serie de valores añadidos que a continuación resumimos.

${ }^{5}$ Síntesis de elaboración propia.

Tendencias Pedagógicas, 33, 2019, pp. 69-82. DOI: 10.15366/tp2019.33.006 
En esencia se trata de una metodología que resulta muy pertinente para investigar casos de colectivos minoritarios o de casos únicos. En esta ocasión, de un menor infractor que se convirtió en educador de menores.

Los investigadores sociales, en la medida de sus posibilidades, deberían tratar de investigar para contribuir a la mejora de la sociedad (Measor y Sikes, 2004). Investigar sobre historias de desigualdad o injusticia estructural parece responder a un compromiso ético en ese sentido. Más aún cuando se trata de personas que se encuentran una posición de poder escaso, casi de invisibilidad (GonzálezMonteagudo, 2010). Las historias de vida en estos casos sirven para restituir a las personas en el espacio social, por lo que tiene un carácter político (González y Padilla, 2014). Hacerles de altavoz quiere poner sobre la mesa un problema que atañe a todos, no sólo a quienes afecta de manera directa. La sociedad será tan avanzada como lo sea la persona que se encuentre en peor situación. Y en ese sentido, pretende hacer pedagogía al respecto y estimular una conciencia social comprensiva y una actitud proactiva con respecto a dichas situaciones, a partir de un conocimiento holístico de esas realidades.

A continuación, se van a detallar todas las fuentes de información y técnicas de recogida de datos y, posteriormente, se compartirán los detalles más básicos del proceso de organización y análisis de los datos.

\subsection{Fuentes de información y técnicas de recogida de datos.}

El trabajo de campo se realizó siguiendo directrices de diversidad, triangulación y saturación para garantizar la rigurosidad y exhaustividad del estudio. A continuación, se relacionan todas las fuentes de información y técnicas de recogida de datos utilizadas para llevar a cabo la investigación.

\subsubsection{Entrevistas biográficas}

Las entrevistas biográficas se realizaron siguiendo las directrices de Pujadas (2002), es decir, se plantearon como un "diálogo abierto con pocas pautas, en el que la función básica del entrevistador es estimular al sujeto analizado para que proporcione respuestas claras" (p. 66). En esta investigación la pregunta de partida que se realizaba en ese sentido era: '¿Cuéntame tu vida?', en el caso de que se estuviese entrevistando a Samir, o ‘¿Cuénteme la vida de Samir?’, en los casos en los que se entrevistaba a personajes secundarios. Una vez se había hecho un recorrido general por su historia, se iba concretizando sobre aspectos específicos vinculados con el foco de investigación.

Se realizaron un total de 5 entrevistas biográficas a Samir y una a cada uno de los doce informantes clave. Todos ellos significativos en el transcurso de la historia objeto de estudio, aportando información relevante para conformar la biografía de Samir. Varios de ellos jugaron un doble rol en dicha historia y fueron entrevistados en consecuencia. Por ejemplo, la trabajadora social del centro de internamiento de menores infractores (CIMI), también pertenecía a la junta directiva de la asociación donde él consiguió su actual empleo en el sistema de protección, por lo que fue entrevistada en virtud de sendos roles en la vida de Samir. Jugaron ese doble rol todos los informantes que aparecen en la siguiente tabla, donde se indica "Posteriormente". Y, por tanto, fueron entrevistados en virtud de ese papel dual. 
Tabla 1

Entrevistas biográficas

\begin{tabular}{|c|l|}
\hline $\begin{array}{c}\text { No de entrevistas } \\
\text { biográficas }\end{array}$ & Descripción Informantes \\
\hline 5 & Samir \\
\hline 1 & Hermano Samir \\
\hline 1 & La jueza que llevó el caso. \\
\hline 1 & Subdirector del Centro de Internamiento de Menores Infractores (CIMI). \\
\hline 1 & $\begin{array}{l}\text { La trabajadora social del CIMI. Posteriormente, jefa de Samir en la asociación de } \\
\text { protección de menores en la que actualmente trabaja. }\end{array}$ \\
\hline 1 & El tutor de Samir en el CIMI \\
\hline 1 & Educadora en el CIMI. Posteriormente, miembro de un Equipo Técnico \\
\hline 1 & $\begin{array}{l}\text { Trabajadora del servicio doméstico del CIMI. Posteriormente, su madre de } \\
\text { acogida }\end{array}$ \\
\hline 2 & A 2 monitores de los talleres del CIMI. Posteriormente amigos suyos. \\
\hline 1 & La tutora de Libertad Vigilada. \\
\hline 1 & Un educador de Libertad Vigilada. \\
\hline 1 & Compañero de trabajo, piso y amigo de Samir. \\
\hline
\end{tabular}

Fuente: elaboración propia.

\subsubsection{Un biograma y un sociograma}

Se elaboraron tras las primeras entrevistas y fueron complementados con entrevistas a Samir para clarificar y contrastar con él la información.

\subsubsection{Herramienta DAFO}

Se utilizó esta herramienta con cada uno de los informantes 7 . En este caso, se realizó el DAFO poniendo el foco en el proceso de reforma juvenil vivenciado por nuestro protagonista.

\subsubsection{Registro iconográfico}

Incluyó más de 200 fotografías. La mayoría de ellas de su periodo en el centro de internamiento. Además, se realizó una entrevista a Samir para que aclarara el significado de dichas instantáneas.

\subsubsection{Un manuscrito redactado por Samir}

El manuscrito tenía como título: "El sentido de mi trabajo", que elaboró voluntariamente para explicar por qué le gustaba su trabajo en el sistema de protección de menores y qué significaba para él desempeñar aquella labor en relación a los delitos que había cometido durante su adolescencia. Tras entregarnos aquel escrito se le realizó una entrevista para profundizar al respecto.

\subsubsection{Análisis documental}

a) Dos expedientes sobre Samir. Permitieron extraer información muy valiosa, desde la perspectiva institucional, y venían a contrastar o complementar el compendio de testimonios recabados:

- El expediente de Samir en el Juzgado de Menores que llevó su caso.

- El expediente de Samir en la Dirección General de Reforma Juvenil.

b) Un informe de seguimiento elaborado por su tutor en el CIMI que no constaba en ninguno de los dos expedientes anteriores.

\footnotetext{
${ }^{6}$ Esta señora era una mujer que trabajaba en la lavandería del centro de internamiento en el que estuvo nuestro protagonista. Samir y ella establecieron una relación fraternal tal, que cuando el joven Almasi tenía que dejar el centro de internamiento y pasar a libertad vigilada ella lo acogió formalmente para facilitar la realización de dicho trámite.

${ }^{7}$ El acrónimo DAFO responde a los siguientes conceptos: Debilidad, Amenaza, Fortaleza y Oportunidad.
} 
c) La legislación vigente que rigió el caso. Concretamente, la Ley Orgánica 5/2000, reguladora de la responsabilidad penal del menor.

\subsubsection{Cuaderno de los investigadores}

Donde se hicieron registros de todo tipo durante el trabajo de campo y se incorporaron las sensaciones de los investigadores durante el proceso.

\subsection{Procesamiento de los datos}

El relato biográfico de Samir fue escrito siguiendo el modelo de relatos cruzados, y teniendo como referentes a su creador Lewis (1973) y Gamella (1990) como máximo exponente en España. Dicho modelo, se articula a partir del cruce de las distintas informaciones registradas para materializar de manera poliédrica, rigurosa, exhaustiva y con sentido la historia que se va a analizar. En el caso de Samir, el cruce se realizó de manera paralela. En primer lugar se construyó su historia en primera persona, es decir, a partir, únicamente, de los datos recabados a través de sus entrevistas biográficas y fotografías. Y justo a continuación, se volvía a contar la historia desde el principio, a partir del resto de testimonios (entrevistas, DAFO, documentos, fotografías, etc.).

Aquel ingente conjunto de información supuso una cantidad de trabajo enorme, sobre todo, a la hora de organizarla. Tanto la categorización como el análisis de los datos se realizaron de manera emergente. Teniendo presente el foco principal de la investigación ${ }^{8}$ se fueron extrayendo y sintetizando los datos hasta conseguir construir su biografía y, posteriormente, armar un listado de categorías significativo y coherente. Los informes conformaron un mosaico ilustrativo de aquella realidad, a través de los cuales se podía ver lo que pasaba por dentro, cual si de una resonancia médica (en este caso sociopedagógica) se tratara. Por lo que se podía tener una visión pormenorizada y de conjunto, a la misma vez (Waller y Simmons, 2009), de la biografía sujeta a estudio.

\section{Resultados: Interpretación y análisis de los datos}

Con algo más de 16 años el joven Almasi empezó a trabajar para la mafia en España. Tarea que desempeñó durante más de un año. Durante ese espacio de tiempo estuvo viviendo de acuerdo a las posibilidades que le brindaba aquella ocupación (disfrutaba de importantes cantidades de dinero, casas de lujo, coches de alta gama, fiestas y cierto desenfreno), sin embargo, diversos profesionales que trabajaron en su proceso de reforma juvenil (todos ellos con dilatada experiencia) y personas varias que lo conocieron en otros contextos tras dicho proceso coinciden en que no tenía un "perfil delictivo":

"Yo a este niño nunca lo vi, nunca, nunca, nunca, la verdad, nunca lo vi un traficante, la verdad, nunca, nunca" [Trabajadora social del Centro de Internamiento de Menores Infractores (CIMI), Biografía, p. CCXVII].

"Que sí, que había sido un delincuente, pero que perfil delictivo no le veía. Tenía unos principios" (Educadora del CIMI, Biografía, p. CCXXVIII).

"A mí Samir no me dio la sensación de tener un perfil delincuencial, en principio todo lo contrario, por lo menos lo que yo viví con él ¿no? No era un chaval que estuviera metiéndose en problemas, ni estuviera metiéndose en broncas. Yo veía que en un momento dado se podía entender el tráfico como manera de supervivencia, tampoco no sé qué tipo de vida tenía allí en Marruecos" (Tutora de Libertad Vigilada, Biografía, p. CCLXXXIII).

“[...] no es un niño con un perfil delictivo” (Jueza que llevó su caso, Biografía, p. CCX).

\footnotetext{
${ }^{8}$ El foco de la investigación constaba de dos partes: Por un lado, se quería indagar sobre cuáles habían sido las claves que provocaron el cambio en la vida de Samir. Y, por otro lado, se quería saber qué había tenido de "educativo" el proceso de reforma juvenil. 
¿Cómo es posible que tras dedicarse durante todos aquellos meses a realizar actividades ilegales casi todas las personas que le conocían, muchas de ellas profesionales que participaron en su proceso de reforma, consideraban que no tenía un perfil delictivo y que no era un delincuente?

Parece que se le consideraba de aquella manera porque mostraba una base socializadora y unos códigos sociales y lingüísticos pacíficos y respetuosos que coincidían con los extendidos y aceptados mayoritariamente. El joven Almasi, a diferencia del conjunto de chicos del centro de internamiento de menores infractores (CIMI), que solían mostrar unas pautas sociales que no se ajustaban a las mayoritarias, se relacionaba con naturalidad fuera de su contexto de origen, tenía buen trato y utilizaba formas adecuadas hacia los demás.

\begin{abstract}
"Samir es particular porque manifiesta una base de moral y de normas que es muy distinta a lo que generalmente se presenta [en el CIMI]. Generalmente los chavales que vienen aquí requieren asumir una norma que no tienen asumida. El caso de Samir no, en el caso de Samir ya se partía de una posición más alta, es decir, se partía de trabajar con un chico que había metido la pata, pero que tenía todo un sustrato de educación fuerte". (Subdirector del CIMI, Biografía, p. CCXII).

"A mí me dio la impresión, por su manera de ser, porque le había visto algo que en los otros no había visto, me refiero a educación, a saber estar en el sitio, entonces como que no me cuadraba allí dentro [en el CIMI]" (Personal de servicio doméstico del CIMI y, posteriormente, Madre de acogida de Samir, Biografía, p. CCXXXVIII).
\end{abstract}

Según las evidencias, el joven Almasi tenía una base socializadora acorde con la requerida en el centro de internamiento y la cultura dominante en España. Poseer esas claves culturales favorecía sus posibilidades de evolución.

Ese hecho resultaba llamativo y lo diferenciaba de la mayoría de sus compañeros en el centro de internamiento, quienes, mayoritariamente, procedían de contextos de exclusión o de capas sociales oprimidas y, por tanto, provenían de entornos duros y hostiles donde la cultura que les envolvía era del mismo tipo (se puede decir que, prácticamente, de supervivencia, pues parece que había evolucionado simbólicamente para contribuir en la satisfacción de las necesidades más básicas de la naturaleza biológica humana). Como consecuencia, la mayoría de internos en el CIMI poseían unas pautas socioculturales que diferían de manera abrupta de las generalmente demandadas (agresividad, violencia, uso de la fuerza, etc., solían ser habituales). En otras palabras, manejaban unos códigos de interacción muy poco elaborados, cuya adecuación se limitaba, fundamentalmente, a su entorno primario.

\footnotetext{
"Muchas veces estos chiquillos se dejan llevar o les llama la atención las posturas más llamativas, más agresivas, más..., lo que denominamos, más llamativas dentro del currículum delincuencial. Lo que más sobresale es lo más bestia, lo más fuerte, lo más agresivo, lo más violento. En el caso de Samir no, en su caso lo que sobresalía era lo más educado, lo más ordenado y precisamente utilizando esos medios conseguía las cosas que a lo mejor otros utilizando otros métodos más violentos no podían conseguir. Pero si Samir en un momento determinado podía discutir conmigo respecto a la aplicación de una norma, a otro ni se le iba a ocurrir. En el caso de Samir como lo hacía de una manera razonablemente educada pues se discutía y punto" (Subdirector del CIMI, Biografía, CCLXXIV).
}

Nuestro protagonista destacaba por sus "buenos modales" en las relaciones personales. No se postulaba como una persona radical o autoritaria. Aunque pudiera ser vehemente en la defensa de sus ideas, no se vislumbraba entre sus códigos comunicativos el uso de la fuerza o la violencia como herramientas de interacción y desenvolvimiento. Por el contrario, parece que el respeto y el diálogo eran sus armas más destacadas. Esto pone de manifiesto que las formas son tan o más, importantes que los contenidos.

Da la sensación de que nuestro protagonista, también, era mucho más racional que la mayoría de sus compañeros del centro de internamiento, que eran más viscerales y se dejaban llevar por las emociones y pulsiones que generaban las circunstancias. 
“[...] los otros niños eran más impulsivos, pienso esto, actúo y lo hago. Él jamás, él era pienso, pienso, pienso y pienso y finalmente actúo, pero claro «yo no voy a meter la pata por un impulso». No, no, él es un controlador, otros niños no (...), entonces el tío era completamente diferente al resto" (Monitor del CIMI, Biografía, pp. CCXXXVI).

En muchos de sus compañeros de centro no existía, únicamente, una carencia de pautas sociales, sino también de algunas pautas básicas relacionadas con la salud, higiene, alimentación, descanso, etc.

“[...] los niños que entran allí, como educación no tienen en su casa, pues sus habitaciones, sus cosas personales, como que el aseo brilla por su ausencia, los dormitorios,... Si tú les das las sábanas para que hagan las camas, ellos pasan olímpicamente de ese tema, porque ellos están acostumbrados a dormir incluso en el suelo. Hay algunos que han llegado allí que no saben que existen las sábanas para ponérselas a las camas" (Personal de servicio doméstico del CIMI y, posteriormente, Madre de acogida de Samir, Biografía, p. CCXL).

La mayor parte de aquellos chicos habían desarrollado esas pautas de comportamiento, como consecuencia de las vivencias que habían experimentado durante su infancia y/o adolescencia. Por eso, la socialización primaria, que se desarrolla en esas etapas tiene gran trascendencia en la trayectoria de vida de las personas.

El problema es que en los contextos de origen y desarrollo de esos menores la situación resulta tan acuciante que los influjos que reciben para desarrollar dicha socialización les permite interiorizar conocimientos, capacidades, actitudes, valores, etc., que les posibilita desenvolverse adecuadamente en ese entorno, pero les genera problemas fuera de él.

La familia, que suele ser el primer agente socializador de las nuevas generaciones, juega un papel decisivo en el desarrollo de ese proceso de socialización y, por tanto, en la configuración humana de los niños y niñas. Pero no todas las familias socializan en el mismo sentido. En el caso de Samir, su familia fue un factor de protección clave. Tuvo la suerte de nacer y crecer en el seno de una familia estructurada que, a pesar de vivir en unas condiciones difíciles, de pobreza, en la que sufrían muchas carencias materiales y de oportunidades vitales, se preocupó por sembrar en los hijos el respeto entre las personas y por las normas sociales establecidas. Y supieron desarrollar en sus descendientes unos patrones socializadores adecuados para el desenvolvimiento en cualquier entorno.

“[...] había ahí una base. En su familia no es habitual el mundo marginal, había una base de educación y de normas que evidentemente facilita mucho el proceso de reeducación. Es decir, trabajar donde ya hay una base, a empezar a generar todo un esquema normativo inexistente" (Subdirector del CIMI, Biografía, p. CCCXIX).

"Destacando por un nivel educativo alto, modales y actitudes de corrección, valores y normas internas que mantiene de las que se enorgullece y de las que reconoce fruto de la buena educación parental recibida" (Informe inicial de la entidad donde Samir cumplió la Libertad Vigilada, Biografía, p. CCLXXVIII).

Sin embargo, en el caso de la mayoría de sus compañeros del centro de internamiento, los progenitores y demás referentes adultos se habían criado en una situación opresiva, no habían podido socializarse de otra manera ni tenido oportunidades de ser educados, por lo que solían reproducir el mismo patrón. Si no se sabe que se puede hacer de otra manera se tiende a repetir la misma dinámica. 
“[...] el entorno de la familia como que no se han preocupado de esos niños para nada. Entonces, esos niños valores no tienen de ninguna clase, no tienen nada más que la calle y el poder de la supervivencia. Ellos tienen que subsistir y tienen que subsistir de cualquier manera, y le da igual que robando, si traficando, si donde sea" (Personal de servicio doméstico del CIMI y, posteriormente, Madre de acogida de Samir, Biografía, pp. CCXLI-CCXLII).

Es más, en algunos casos los progenitores y/o referentes familiares adultos lo que les enseñaban iba en contra de lo que la sociedad demandaba. Los chicos eran producto de la situación que estaban viviendo o habían vivido.

"Y del que sus padres están en la cárcel porque son estafadores, porque son ladrones, porque es lo que han vivido (...). Tienen mucho desarraigo, mucho, y son dignos de lástima. La gente cuando dices eso se pone las manos en la cabeza. Somos conscientes de que si uno le ha dado un tironazo a una vieja del bolso y le ha abierto la cabeza, la verdad es que no te hace ninguna gracia, pero es que no les han enseñado otra cosa, ni han vivido otra cosa, ni le ha pegado el padre dos tortas por hacer algo que no debía, sino al contrario, «Me has traído esto, mañana tráeme dos. Y como no me lo traigas te harto». Y eso es así, y con mucha falta de cariño, mucha falta de cariño" (Personal de servicio doméstico del CIMI y, posteriormente, Madre de acogida de Samir, Biografía, p. CCXLII).

Por eso, se trata de un problema estructural, de desigualdad, de pobreza extrema, de exclusión, etc. En tanto no se corrijan de raíz esos estados de necesidad (Calderón, 2014) seguirá habiendo contextos donde crezcan personas interiorizando ese tipo de patrones de supervivencia, que tienen un carácter individualista y que, como consecuencia, se alejan de los conciertos sociales.

Una interculturalidad crítica (Ruiz, 2003) debe abogar, por tanto, por una transformación radical de esa situación para que no existan contextos en los que no se puedan satisfacer las necesidades más básicas (Maslow, 1975; López, 2008) y se permita el desarrollo equilibrado y socialmente adecuado de los sujetos, provengan de donde provengan.

Mientras tanto, el sistema educativo obligatorio, que suele ser el segundo agente socializador más importante de la población, debe compensar las carencias de partida que presenten esos niños. En los entornos socioculturales deprimidos, uno de los objetivos prioritarios debe ser tratar de proporcionar las pautas de socialización y los códigos sociolingüísticos necesarios para otorgarles herramientas culturales que les permitan romper con el determinismo y tener otras oportunidades en la vida.

Además, el proceso intercultural requiere de pedagogía social sobre el conjunto de la población (de la parte hegemónica) (Sánchez, Milud, Mohamed, Mohamed y Mohamed, 2017), para, entre otros asuntos, generar comprensión y que se pueda establecer algún tipo de interacción entre las partes. De lo contrario, los estereotipos impedirán un acercamiento y un verdadero diálogo entre ellas (Segura, Alemany y Gallardo, 2016).

"Yo siempre digo que muchas veces la vida, no sabemos lo que hay que hacer para vivir y hay que tener esa capacidad de comprender" (Trabajadora social del CIMI, Biografía, p. CCXVII).

\section{Conclusiones}

La interculturalidad no es sólo diversidad de culturas provenientes de distintos países o sociedades, la interculturalidad también afecta a todo aquello que tiene que ver con la interacción entre personas con distintos patrones y niveles culturales dentro de una misma comunidad.

La socialización experimentada durante la infancia y la adolescencia resulta un elemento clave en la construcción de la persona. En esas etapas del desarrollo se cimentan algunos de los pilares fundamentales que configuran, de manera singular, a cada persona. La enculturización que se desarrolla durante ese proceso proporciona esquemas lingüísticos, cognitivos, sociales, afectivos, morales, etc. que condicionan sustancialmente el desarrollo del individuo (Spindler, 2006). 
La familia es el agente socializador más importante. Asimismo es el primer contexto cultural ya que, en gran medida, el menor comienza a constituirse como persona en virtud de la educación que experimente en su núcleo de protección. Madres, padres, o cuidadores, son referentes para los niños y adolescentes. Son adultos que ejercen una enorme influencia en el proceso de construcción de la identidad de los menores. La interpretación de la realidad de esos referentes, el modo que tienen de entender y materializar: normas, valores, sentimientos, actitudes, etc., condiciona la manera de entender el mundo de las nuevas generaciones. En virtud de ellas, los menores crearán buena parte de su manera de ser y estar en el mundo.

Teniendo ello en cuenta, las familias deben tratar de contribuir a que el niño o adolescente se desarrolle de manera saludable, no sólo biológica, sino, además, cultural y socialmente. Un desarrollo que permita su capacidad de adaptación y desenvolvimiento en cualquier escenario, no sólo en su contexto primario. Con especial énfasis, en los casos de niños y adolescentes originarios de contextos más vulnerables, que suelen manejar unos códigos muy restringidos. De lo contrario, tendrán muy pocas posibilidades de desarrollar su vida de otro modo y, por tanto, se verán empujados a la reproducción social.

El problema es que los progenitores, o adultos de referencia, de los niños y adolescentes que proceden de esos estratos se encuentran muy limitados culturalmente. El escenario social genera una influencia tan enorme que difícilmente les permite hacerlo de otro modo; no saben hacerlo de otra forma, porque no suelen conocer otras maneras de llevarlo a cabo. Por otro lado, las necesidades vitales suelen ser tantas y tan acuciantes que prima la supervivencia. Supervivencia que genera unos patrones de desarrollo que no suelen coincidir en algunos aspectos con las convenciones sociales mayoritarias.

De ahí que el sistema educativo deba redoblar sus esfuerzos en los entornos culturalmente desfavorecidos, porque en esos entornos se hace muy necesario compensar las carencias que de partida presenta ese alumnado, para tratar de romper con el determinismo que les persigue (Leiva, 2011). Además de implementar otros procesos pedagógicos (instructivos, educativos, etc.), se ha de hacer especial hincapié en completar una socialización primaria que les permita ser suficientemente versátiles e inteligentes para acomodarse y salir airosos en cualquier contexto. En ese sentido, se les ha de tratar de hacer culturalmente bilingües (no desde un punto de vista idiomático), es decir, dotarles de una serie de códigos vehiculares en la cultura mayoritaria para que puedan disfrutar de una mayor igualdad de oportunidades. Por supuesto, sin que cause ningún menoscabo a su identidad individual y colectiva.

"Educación y enculturación tienen como base la inclusión, la conjunta interpretación y el consenso del individuo y la sociedad en la que vive; es en esta inclusión y a través de la educación y enculturación donde el individuo construye su identidad" (Gómez, 2014, p.168).

La escuela intercultural crítica es un agente fundamental para el encuentro entre esas culturas (Chamseddine, 2015), entre las trayectorias biográficas de los menores y su proceso de construcción identitaria compartida.

$\mathrm{Y}$ es que hemos visto que algunos lenguajes, sobre todo comportamentales en el caso de los varones del centro de internamiento, resultaban una barrera (Echeita y Ainscow, 2011) para la interacción en otros escenarios. Las formas son tan o más importantes que los contenidos. Guardar las formas públicamente contribuye a obtener una consideración socialmente aceptada. En nuestra sociedad se mira con recelo a quienes se comportan de un modo diferente al mayoritario, especialmente en el caso de las personas procedentes de estratos sociales deprimidos. Sin embargo, debemos ser conscientes de que esas conductas o formas de interacción, aunque puedan resultar abruptas a la mayoría, son lenguajes. Lenguajes producto de la socialización vivida y de las situaciones opresivas a las que han sido sometidos. De hecho, debería haber una conciencia social comprensiva porque más abrupta, agresiva y violenta suele ser la realidad con ellos. Ellos simplemente han desarrollado esas maneras de relacionarse (ese lenguaje, esos 
códigos) a partir de su vivencia en esos escenarios.

Por todo ello, se requiere de una interculturalidad crítica, para transformar las estructuras y dinámicas que generan esas situaciones opresivas y romper el 'statu quo' que impide el desarrollo de una verdadera sociedad inclusiva (Cohen-Emerique, 1989). Lo que pasa por crear escenarios garantizando unas condiciones de vida en las que las necesidades básicas estuvieran cubiertas. Eso posibilitaría una mayor interacción entre las personas de los distintos estratos culturales.

\section{Referencias bibliográficas}

Bernstein, B. (1998). Pedagogía, control simbólico e identidad. Barcelona: El Roure.

Bernstein, B. (1990). Poder, educación y conciencia. Sociología de la transmisión cultural. Madrid: Akal.

Bernstein, B. (1989). Clases, códigos y control. I. Estudios teóricos para una sociología del lenguaje. Madrid: Akal.

Bernstein, B. (1988). Clases, códigos y control. II. Hacia una teoría de las transmisiones educativas. Madrid: Akal.

Calderón Almendros, I. (2014). Educación y esperanza en las fronteras de la discapacidad: estudio de caso único sobre la construcción creativa de la identidad. Madrid: Cinca.

Carr, D. (2005). El sentido de la educación. Una introducción a la filosofía y a la teoría de la educación y de la enseñan₹a. Barcelona: Graó.

Chamseddine, M. (2015). Construcción de identidad compartida en un aula intercultural. Revista Electrónica Interuniversitaria de Formación del Profesorado, 18(3), pp. 69-81.

Cohen-Emerique, M. (1989). Travailleurs sociaux et migrants. La reconnaissance identitaire dans le processus d'aide. En C. Camilleri et M. Cohen-Emerique (dir.). Chocs de cultures: Concepts et enjeux pratiques de linterculturel (77-115). Paris: L'Harmattan.

Echeita Sarrionandía, G. \& Ainscow, M. (2011). La educación inclusiva como derecho. Marco de referencia y pautas de acción para el desarrollo de una revolución pendiente. Revista Tejuelo, 12, pp. 26-46.

Gamella, J. F. (1990). La bistoria de Julián. Memorias de heroina y delincuencia. Madrid: Popular.

Gómez Redondo, C. (2014). Marcos diluidos: mezclas y suspensiones de educación y enculturación. Pulso, pp. 167-186

González, M. F. \& Padilla-Carmona, M. T. (2014). Investigación narrativa: las historias de vida. En B. Ballesteros Velázquez (coord.). Taller de investigación cualitativa (pp.77-102). Madrid: UNED

González-Monteagudo, J. (2010). Biografía, Identidad y Aprendizaje en estudiantes universitarios no tradicionales. Estudio de caso de una mujer trabajadora. Profesorado: Revista de currículum y formación del profesorado, 14(3), pp. 131-147.

Herrera Pastor, D. \& De Oña Cots, J. M. (2017). Personalizar la intervención educativa con menores en situación de riesgo es fundamental para su desarrollo, la igualdad de oportunidades y la justicia social. Revista Internacional de Educación para la Justicia Social, 6(2), pp. 149-165. https://doi.org/10.15366/riejs2017.6.2.009

Herrera Pastor, D. \& De Oña Cots, J. M. (2016). La importancia del ambiente de aprendizaje. Pedagogía ecológica en un centro de menores. Revista Fuentes, 18(1), pp. 77-90. DOI: http://dx.doi.org/10.12795/revistafuentes.2016.18.1.05

Herrera Pastor, D. (2016). Aportaciones metodológicas a la investigación biográfica, relacionadas con el procesamiento y la organización de los datos. Cuestiones Pedagógicas, 25 (2015/2016), pp. 145-160. DOI: http://dx.doi.org/10.12795/CP.2016.i25.11

Herrera Pastor, D., Mancila, I. \& Kushner, S. (2017). A cross-narrative analysis in biographical inquiry. A research note. The New Educational Review, 48(2), pp. 285-296. DOI: 10.15804/tner.2017.48.2.23

Leiva Olivencia, J. J. (2015). Las esencias de la educación intercultural. Málaga: Aljibe.

Leiva Olivencia, J. J. (2013). Convivencia y educación intercultural. Análisis y propuestas pedagógicas. Alicante: ECU.

Leiva Olivencia, J. J. (2011). La educación intercultural: un compromiso educativo para construir una escuela sin exclusiones, Revista Iberoamericana de Educación, 56, pp. 1-14.

Lewis, O. (1973). Los hijos de Sánchez: autobiografía de una familia mexicana. México: Joaquín Mortiz.

Londoño Vásquez, D. A. \& Castañeda Naranjo, L. S. (2011). Basil Bernstein y la relación lenguaje-

Tendencias Pedagógicas, 33, 2019, pp. 69-82. DOI: 10.15366/tp2019.33.006 
educación: el caso del Semestre de Afianzamiento (SEA) de la Institución Universitaria de Envigado. Revista Lasallista de Investigación, 8(2), julio-diciembre, pp. 18-32.

López Herrerías, J.A. (2013). Etimologias Pedagógicas. Madrid: Editorial CCS.

López Sánchez, F. (2008). Necesidades en la infancia y en la adolescencia. Respuesta familiar, escolar y social. Madrid: Pirámide.

Maslow, A. (1975). Motivación y personalidad. Barcelona: Sagitario.

Maturana Romesin, H. (1997). Emociones y lenguaje en educación y política. Chile: Dolmen Ediciones / Gránica.

Measor, L. \& Sikes, P. (2004). Una visita a las historias de vida. Ética y metodología de la historia de vida. En I.F. Goodson (ed.). Historias de vida del profesorado (269-295). Barcelona: Octaedro.

Poveda, D. (2001). La educación de las minorías étnicas desde el marco de las continuidadesdiscontinuidades familia-escuela. Gazeta de Antropología, 17, pp. 17-31.

Pujadas Muñoz, J. J. (2002). El método biográfico: El uso de las historias de vida en ciencias sociales. Madrid: Centro de Investigaciones Sociológicas.

Ruiz Román, C. (2003). Educación intercultural: una visión crítica de la cultura. Barcelona: Octaedro.

Sánchez-Fernández, S., Milud-Ahmed, A., Mohamed-Abdel-lah, A., Mohamed Abdelkader, N., \& Mohamed-Mohamed Berkan, N. (2017). Los menores extranjeros no acompañados de Melilla. Análisis de su situación y propuesta de formación socioeducativa. MODULEMA, 1, pp. 121142.

Sarramona, J. (2008). Teoría de la Educación. Reflexión y normativa pedagógica. Barcelona: Ariel.

Segura Robles, A., Alemany Arrebola, I. \& Gallardo Vigil, M.A. (2016). Electronic Journal of Research in Educational Psychology, 14(2), pp. 393-416. http://dx.doi.org/10.14204/ejrep.39.15069

Spindler, G. (2006). La transmisión de la cultura. En H. M. Velasco Maillo, F. J. García Castaño \&, A. Díaz de Rada (Eds.), Lecturas de Antropología para educadores. El ámbito de la antropología de la educación y de la etnografía escolar, (pp.205-241) Madrid: Trotta.

Waller, R. \& Simmons, J. (2009). Vidas a través de la lente de un ojo de buitre: Interpretando cuentos de aprendices. En J.I. Rivas Flores \& D. Herrera Pastor (coords.). Vozy educación. La narrativa como enfoque de interpretación de la realidad (pp.55-74). Barcelona: Octaedro. 